\title{
Inhibition of iNOS activity enhances the anti-tumor effects of alpha-galactosylceramide in established murine cancer model
}

\author{
Hiroyasu Ito ${ }^{1}$, Tatsuya Ando ${ }^{1}$ and Mitsuru Seishima ${ }^{1}$ \\ ${ }^{1}$ Department of Informative Clinical Medicine, Gifu University Graduate School of Medicine, Yanagido, Gifu, Japan \\ Correspondence to: Hiroyasu Ito, email: hito@gifu-u.ac.jp \\ Keywords: cancer immunotherapy, alpha-garactosylceramide, induced nitric oxide synthase, tumor antigen-specific immune \\ response, MDSC \\ Received: May 22, $2015 \quad$ Accepted: October 06, $2015 \quad$ Published: October 19, 2015
}

This is an open-access article distributed under the terms of the Creative Commons Attribution License, which permits unrestricted use, distribution, and reproduction in any medium, provided the original author and source are credited.

\section{ABSTRACT}

Alpha-garactosylceramide (GaICer) has been shown to have anti-tumor effect in the basic research and clinical studies. However, anti-tumor effect of GalCer is limited. The administration of GalCer increases the production of IFN-Y which is involved in the suppression of tumor growth. On the other hand, the enhancement of IFN-Y production increases immunosuppressive factors such as nitric oxide. This suppressive action might impair the anti-tumor effect of GalCer. In the present study, we evaluated the anti-tumor effect of GalCer in the absence of inducible nitric oxide synthase (iNOS). In lung metastatic model, the number of tumor nodules in the lung of iNOS-KO mice treated with GalCer was significantly reduced compared with that of WT mice treated with GalCer. Moreover, L-NAME, which is the inhibitor for iNOS, enhanced the anti-tumor effect of GalCer in lung metastatic model. The frequency of CD8+ cells in bronchoalveolar lavage fluid increased in iNOS-KO mice treated with GalCer. The administration of GalCer increased the frequency of myeloidderived suppressor cells (MDSCs) in the lung from tumor-bearing WT mice, but the increase of MDSCs in the lung was not induced in iNOS-KO mice. The subcutaneous tumor experiments revealed that the administration of GalCer in the absence of iNOS expression significantly enhanced the induction of tumor antigen-specific response. Finally, our results indicated that the inhibition of iNOS expression could enhance the therapeutic efficacy of GalCer via the increase of tumor antigen-specific immune response and the suppression of MDSCs.

\section{INTRODUCTION}

Alpha-galactosylceramide (GalCer) is identified as the ligand of Va14+ natural killer (NK) T cells. Va14+ NKT cells distinct from mainstream $T$ cells, $B$ cells and NK cells have been identified. These cells are found in relative abundance in tissues such as spleen, bone marrow, thymus, and liver, and are characterized by the co-expression of NK cell receptors and invariant $\mathrm{T}$ cell receptors encoded by V $\alpha 14$ and J $\alpha 18$ gene segments [1]. Many reports previously demonstrated that the administration with GalCer induces the anti-tumor activity via the activation of NKT cells. The activated NKT cells can secrete various cytokines, and these cytokines contribute to the GalCer-induced anti-tumor effect in vivo [2-6]. However, the administration with GalCer alone is not so effective. Therefore, several reports evaluated the anti-tumor effect of GalCer by the combination with IL-12 or IL-18 [7, 8].

Inducible nitric oxide synthase (iNOS) is an enzyme that produces nitric oxide (NO) in several situations. In particular, NO promotes angiogenesis, metastasis, and immunosuppression in tumor microenvironment [9]. Various tumor cells can induce NO production via the up-regulation of iNOS expression, and iNOS expression is involved in the prognosis of the patient with any cancer $[10,11]$. Previous studies demonstrated that myeloid-derived suppressor cells (MDSCs) also produce $\mathrm{NO}$ and suppress the host immune response in tumor microenvironment [12]. Thus, NO production contributes to the progression of cancer and it might be critical to suppress the expression of iNOS for cancer 
immunotherapy. Recent reports examined that the administration of GalCer enhanced the iNOS expression in EAE model [13]. The co-administration with GalCer and toll like receptor (TLR) agonist extremely enhanced NO production $[14,15]$. Thus, the activation of NKT cells is effective for anti-tumor immunity via various cytokines, but is counteracted by the simultaneous induction of iNOS which has immunosuppressive effect in tumor-bearing animals. In the present study, we addressed the hypothesis that the inhibition of iNOS activity during cancer therapy using GalCer will enhance the tumor antigen-specific host immune response to inhibit tumor growth. We were able to show that the administration of GalCer and simultaneous inhibition of iNOS activity promote the tumor antigenspecific immune response, leading to the suppression of established lung metastasis and subcutaneous tumor model in vivo.

\section{RESULTS}

\section{Up-regulation of iNOS expression after the administration with GalCer}

GalCer have been recently used for cancer therapy in basic and clinical research. Although the administration with GalCer enhances the host immune response, immunosuppressive factors, including iNOS, are simultaneously induced by GalCer. We first examined the iNOS expression in lung of B16 F10 cells-bearing mice after intraperitoneal injection with GalCer. As shown in
Figure1A, iNOS mRNA expression was increased in the lung of tumor-bearing WT mice after GalCer injection $(P<0.05)$. We next examined the mRNA expression of iNOS in CD11b+ cells of tumor-bearing WT mice (Figure 1B). CD11b+ cells were magnetically collected from bronchoalveolar lavage fluid (BALF) of tumor-bearing mice by MACS system. The iNOS mRNA expression of $\mathrm{CD} 11 \mathrm{~b}+$ cells in BALF was extremely up-regulated by the administration with GalCer $(P<0.05)$.

\section{Anti-tumor effect of GalCer was enhanced by the suppression of iNOS activity in lung metastasis models}

B16F10 cells and C26 cells were intravenously injected to WT or iNOS-KO mice to establish lung metastasis models. Tumor nodules in the lung could be confirmed at 5 days after the administration with tumor cells (data not shown). GalCer $(2 \mu \mathrm{g} / \mathrm{mouse})$ was intraperitoneally administered into B16F10 cells-bearing WT and iNOS-KO mice 7 days after the inoculation of tumor cells. Seven days after GalCer injection, mice were killed, and their lungs were removed to count superficial metastatic nodules (Figure 2A). The number of nodules in the lung was significantly reduced in iNOS-KO mice treated with GalCer $(P<0.05)$. $\mathrm{N}^{\mathrm{G}}$-nitro-L-arginine methyl ester (L-NAME) is an iNOS inhibitor; hence, we used this agent to substantiate data obtained from iNOS-KO mice. WT mice were orally administered with L-NAME at 0 or 2 $\mathrm{mg} / \mathrm{ml}$ in drinking water for 1 day before GalCer injection. The co-administration with GalCer and L-NAME

B

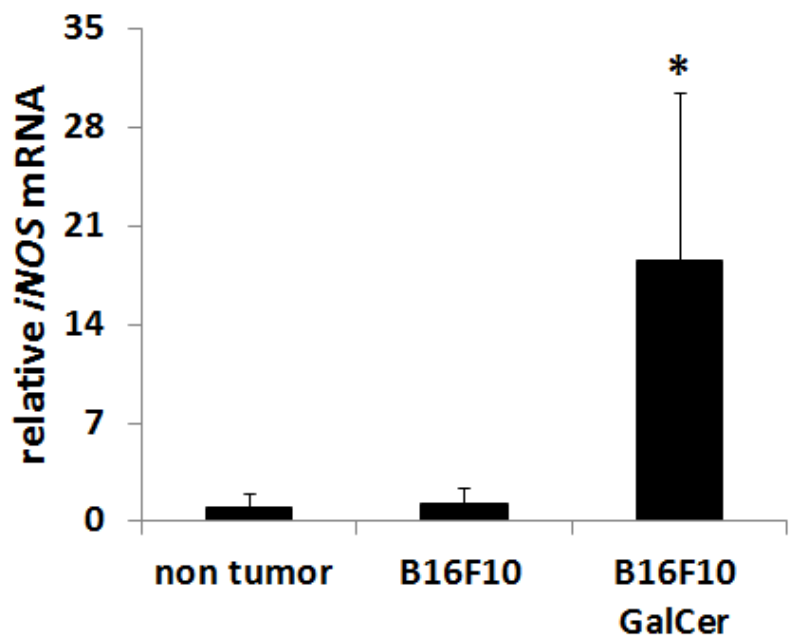

Figure 1: Up-regulation of iNOS expression after GalCer administration in tumor-bearing mice. $\mathrm{B} 16 \mathrm{~F} 10$ cells $\left(3 \times 10^{5} /\right.$ mouse) were intravenously administered to WT mice. WT mice were intraperitoneally injected with GalCer $(2 \mu \mathrm{g} / \mathrm{mouse})$ at $7 \mathrm{days}$ after the inoculation of tumor cells. A. The relative expression levels of iNOS mRNA in the lung of tumor-bearing mice treated with GalCer were measured by real-time RT-PCR. B. CD11b+ cells were magnetically isolated from BALF, and iNOS mRNA expression of CD11b+ cells were measured by real-time RT-PCR. The results were normalized to the expression of 18S rRNA. Each value is shown as mean and SEM for three mice. * indicates statistically significant differences. 
significantly decreased the number of tumor nodules in the lung $(P<0.05)$ (Figure $2 \mathrm{~B})$. Next, we examined the effect of GalCer and L-NAME on the different lung tumor model. C26 cells were inoculated to WT mice, and GalCer and L-NAME were administered into C26 tumor-bearing mice. The co-administration with GalCer and L-NAME significantly reduced the number of tumor nodules in C26 lung tumor model $(P<0.05)$ (Figure $2 \mathrm{C})$.
A

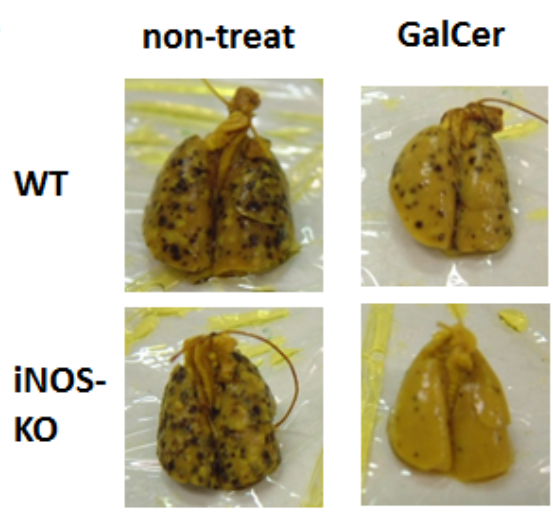

B

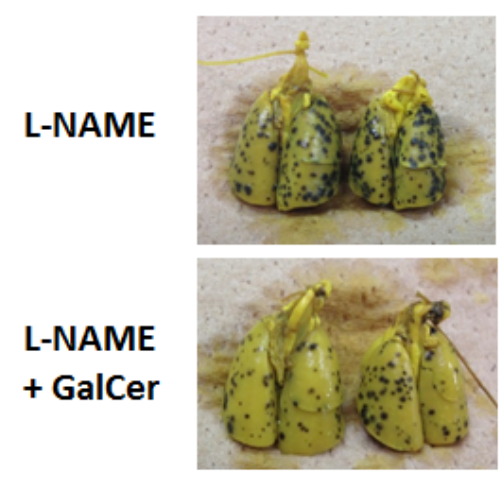

C

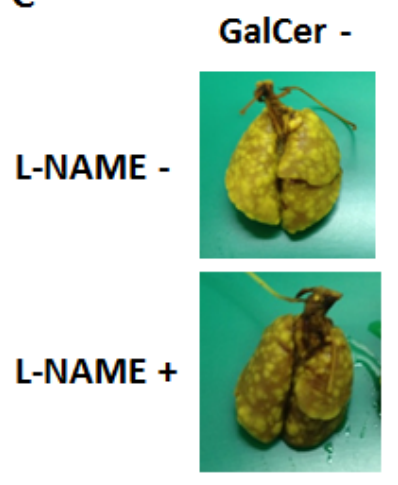

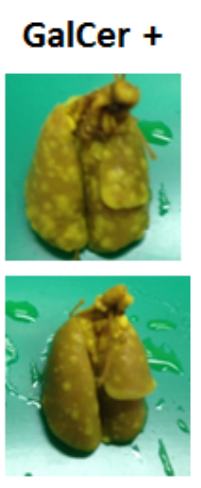
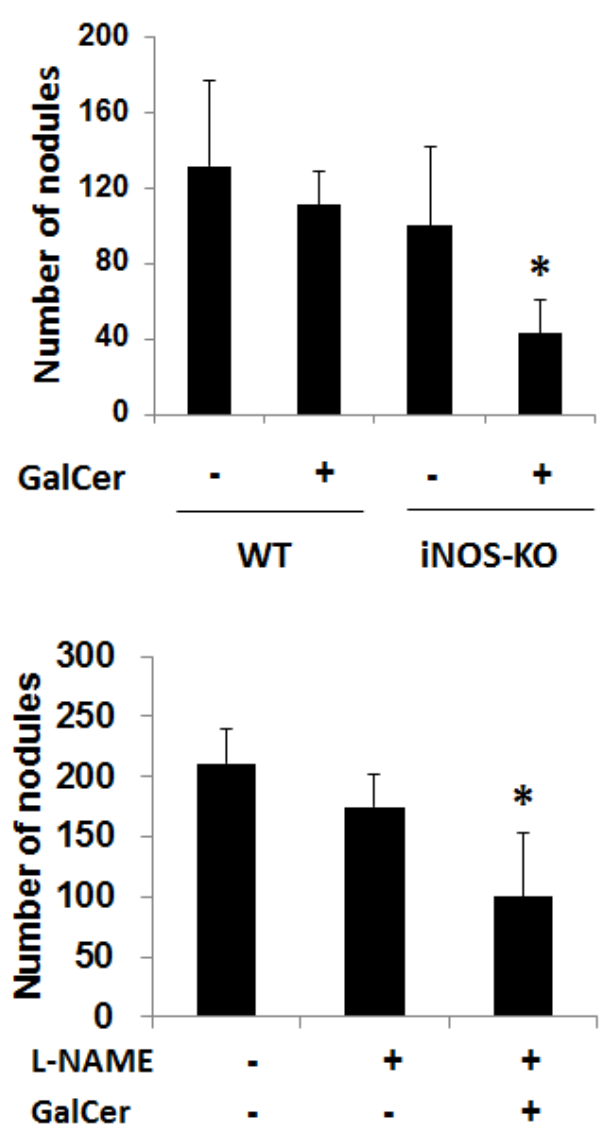

Figure 2: Inhibition of iNOS expression enhanced the anti-tumor effect of GalCer on lung metastatic cancer model. A. B16F10 cells $\left(3 \times 10^{5} /\right.$ mouse $)$ were intravenously administered to WT and iNOS-KO mice on day 0 . Tumor-bearing mice were intraperitoneally injected with GalCer $(2 \mu \mathrm{g} /$ mouse $)$ at 7 days after the inoculation of tumor cells. Mice were killed at 7 days after the injection of GalCer, and their lungs were removed to count superficial metastatic nodules. The number of superficial metastatic lung tumor was significantly decreased in iNOS-KO mice treated with GalCer $(P<0.05)$. B. B16F10 cells $\left(3 \times 10^{5} /\right.$ mouse $)$ were intravenously administered to WT mice on day 0 . WT mice were administered with L-NAME orally at $2 \mathrm{mg} / \mathrm{ml}$ in drinking water for 1 days before and 7 days after GalCer injection. Mice were killed at 7 days after the injection of GalCer. The number of superficial metastatic lung tumor was significantly reduced in mice with L-NAME and GalCer $(P<0.05)$. C. CT26 cells $\left(2 \times 10^{5} / \mathrm{mouse}\right)$ were intravenously administered to WT mice on day 0 . The mice were orally administered L-NAME in drinking water at 6 days after tumor injection, and intraperitoneally injected with GalCer 1 day later. Mice were killed at 7 days after the injection of GalCer. The number of superficial metastatic lung tumor was significantly reduced in mice with L-NAME and GalCer $(P<0.05)$. Each value is shown as mean and SEM for 4-6 mice. 
Effect of GalCer treatment on the phenotype of lymphocyte in BALF from tumor-bearing WT and iNOS-KO mice

We next measured the frequency of $\mathrm{CD} 4+, \mathrm{CD} 8+$, and $\mathrm{CD} 11 \mathrm{~b}+/ \mathrm{Ly} 6 \mathrm{G}+$ cells in BALF of tumor-bearing WT and iNOS-KO mice at 3 day after GalCer administration. As shown in Figure 3A, the frequency of CD8+ cells was significantly increased in iNOS-KO mice treated with
GalCer. On the other hand, the rate of $\mathrm{CD} 11 \mathrm{~b}+/ \mathrm{Ly} 6 \mathrm{G}+$ cells in iNOS-KO mice treated with GalCer was decreased compared to that in WT mice treated with GalCer. There was no difference between WT and iNOS-KO mice treated with GalCer in the ratio of CD4+ cells. Moreover, CFSE-based $\mathrm{T}$ cell suppression assay revealed that the proliferation of CFSE-labeled CD3+ cells co-cultured with BALF cells from GalCer-treated WT mice was more suppressed compared with those from non-treated WT mice or GalCer-treated iNOS mice (Figure 3B).

A
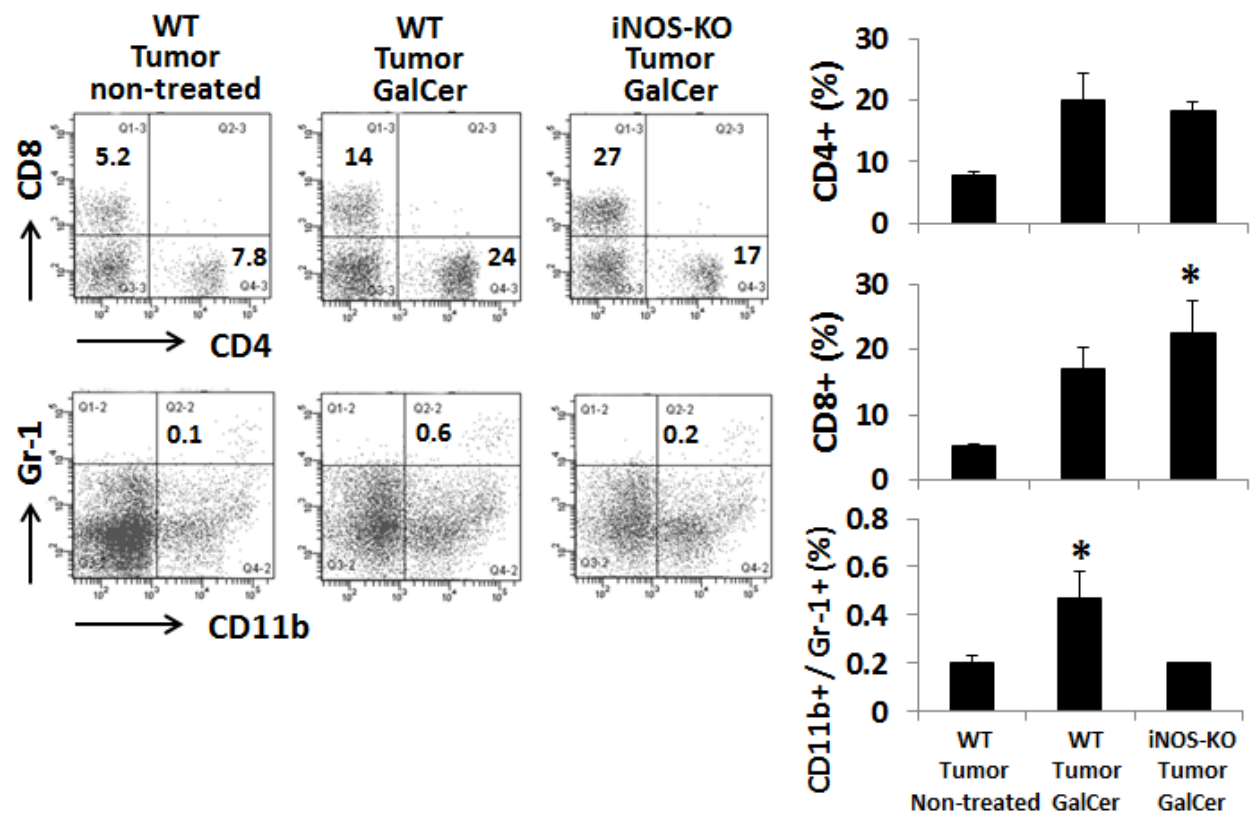

B
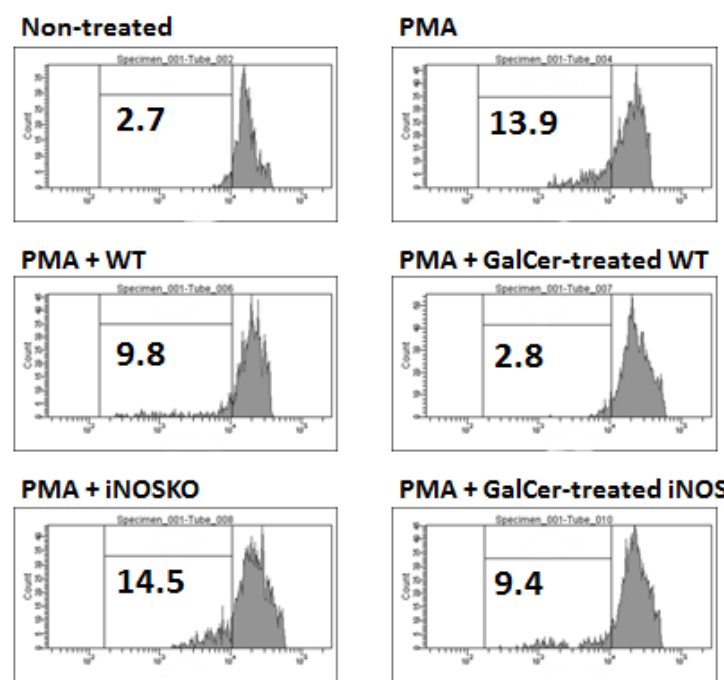

PMA + GalCer-treated iNOSKO

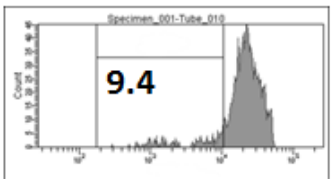

Figure 3: Changes of the proportion of CD8+ and CD11b+/Ly6G+ cells in BALF cells from tumor-bearing WT and iNOS-KO mice treated with GalCer. The lymphocytes in BALF from tumor-bearing WT and iNOS-KO mice were isolated 3 days after GalCer administration. A. Data show the percentage of CD4+, CD8+, and CD11b+/Ly6G+ cells. Each data point and error bar represent the mean and SEM, respectively, of data from triplicate samples. * indicates statistically significant differences. Closed bar; WT mice, open bar; iNOS-KO mice. B. BALF cells were isolated from WT and iNOS-KO mice administered with GalCer. Naïve splenocytes labeled with CFSE were co-cultured with BALF cells in 96-well plates at a cell-count ration of 1/1 for 3 days in the presence of PMA. Data were representative of at least three independent experiments with similar result. 
IFN- $\gamma$, FasL, CCL2, and CXCL9 mRNA expression in the lung of tumor-bearing WT and IDO-KO mice treated with GalCer

IFN- $\gamma$ and FasL are critical on the establishment of anti-tumor immunity. Moreover, CCL2 and CXCL9 are involved in the chemoattractant of $\mathrm{T}$ cells $[16,17]$. Next, we measured mRNA levels for IFN- $\gamma$, FasL, CCL2, and CXCL9 in the lung from tumor-bearing WT and iNOS-KO mice after GalCer administration (Figure 4). The mRNA expression of IFN- $\gamma$, FasL, and CXCL9 significantly increased in iNOS-KO mice at 3 days after GalCer administration. Although the expression of CCL2 mRNA was up-regulated in WT and iNOS-KO mice after the administration with GalCer, there was no difference between WT and iNOS-KO mice in the expression of CCL2 mRNA after GalCer injection (Figure 4).
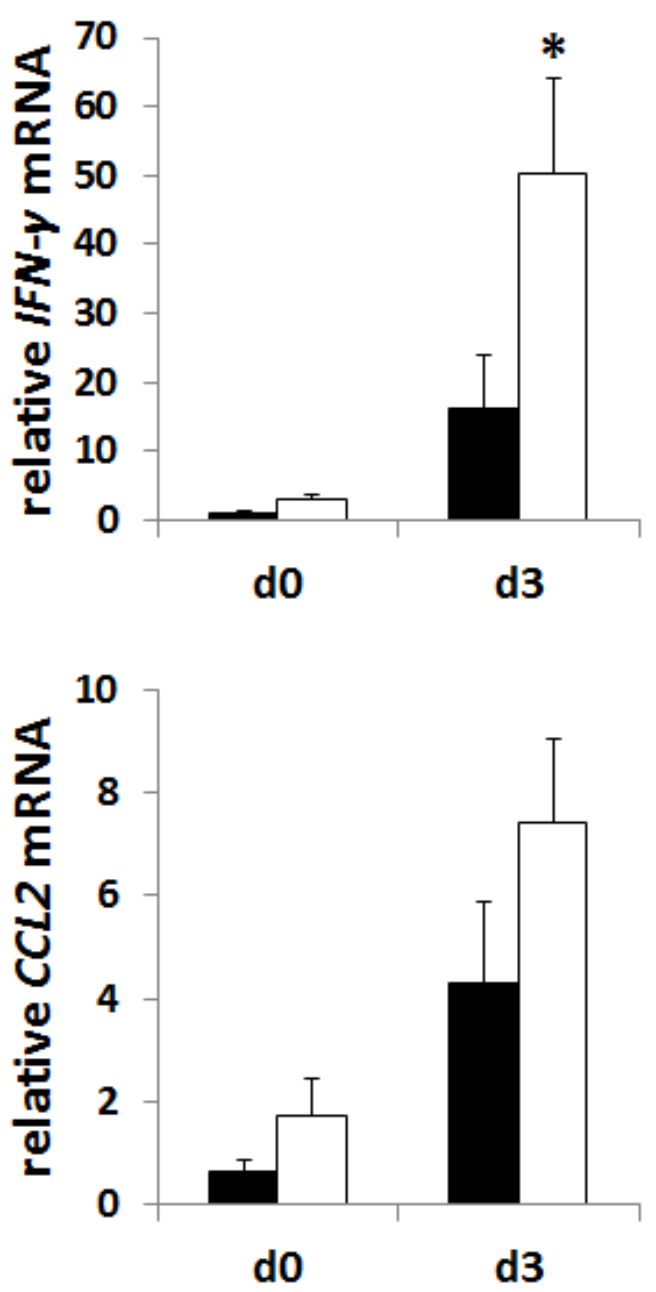

\section{Effect of GalCer injection on subcutaneous primary tumors in WT and iNOS-KO mice}

We next examined the anti-tumor effect of GalCer in WT and iNOS-KO mice using subcutaneous tumor models. EG7 cells were inoculated in to the right flank of WT and iNOS-KO mice, and GalCer was intraperitoneally injected when the tumor size grew to approximately $5 \mathrm{~mm}$ in diameter. The administration with GalCer significantly impaired the growth of subcutaneous tumor in iNOS-KO mice $(P<0.05)$ (Figure 5A). To clarify the mechanism by which GalCer injection promotes anti-tumor effect in iNOS-KO mice, cells were isolated from tumor DLN of mice bearing subcutaneous EG7 tumor. The DLN cells were cultured ex vivo with the OVA SIINFEKL peptide, which is a CD8-restricted epitope expressed by EG7. DLN cells from tumor-bearing WT and iNOS-KO mice
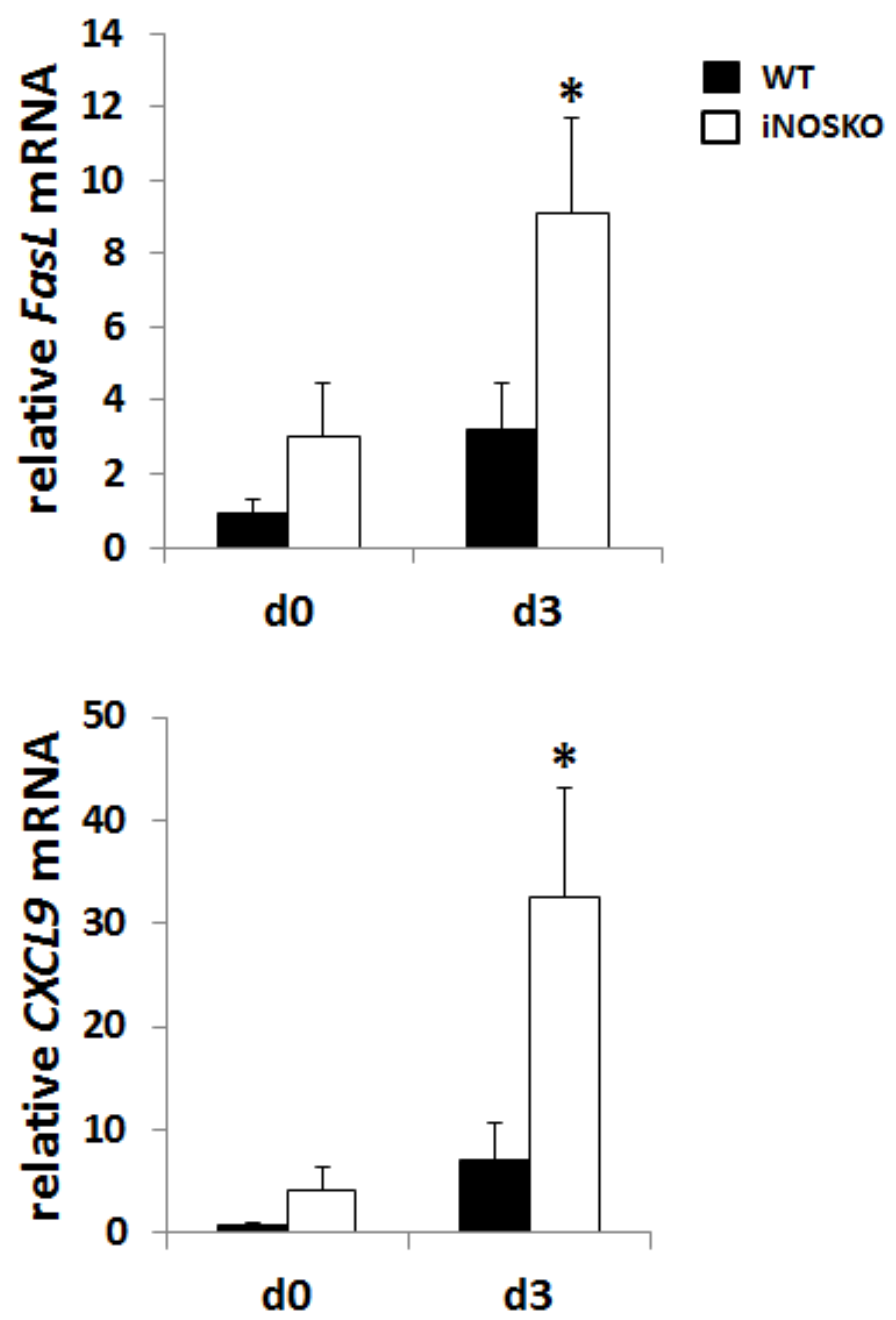

Figure 4: The mRNA expression of IFN- $\gamma$, FasL, and CXCL9 in the lung from tumor-bearing iNOS-KO mice was up-regulated after GalCer administration. B16F10 cells $\left(3 \times 10^{5} /\right.$ mouse) were intravenously administered to WT mice on day 0 . WT mice were intraperitoneally injected with GalCer at day 7 after the inoculation of tumor cells. The relative expression levels of IFN- $\gamma$, FasL, CCL2, and CXCL9 mRNA in the lung of WT and iNOS-KO mice treated with GalCer were measured using real-time RT-PCR. The results were normalized to the expression of 18S rRNA. Each data point and error bar represent the mean and SEM, respectively, of data from triplicate samples. * indicates statistically significant differences. 
treated with GalCer responded to stimulation by OVA peptide by secreting IFN- $\gamma$. As shown in Figure $5 B$, the number of spot significantly increased by the stimulation with OVA peptide in DLN cells from iNOS-KO mice treated with GalCer $(P<0.05)$. Moreover, we examined the EG7-specific lysis caused by CD8+ T cells in DLN cells from WT and iNOS-KO mice treated with GalCer $e x$ vivo (Figure 5c). Specific cytotoxicity of CD8+ T cells to EG7 cells in DLN cells from the iNOS-KO mice treated with GalCer was significantly increased compared to that from WT mice treated with GalCer $(P<0.05)$. We measured the expression of IFN- $\gamma$ mRNA in DLN of WT and iNOS-KO mice at 3 days after GalCer administration (Figure 5D). The expression of IFN- $\gamma$ mRNA in iNOS$\mathrm{KO}$ mice was significantly increased after GalCer administration compared with that in WT mice. In the tumor, the frequency of CD8+ cells in iNOS-KO mice was significantly increased after GalCer administration compared to that in WT mice $(P<0.05)$ (Figure 6A). On the other hand, the frequency of CD11b+/Ly6G+ cells in the tumor of iNOS-KO mice treated with GalCer decreased compared to that of WT mice $(P<0.05)$. In CFSE-based $\mathrm{T}$ cell suppression assay, the proliferation of CFSE-labeled CD3+ cells co-culture with CD11b+ cells from GalCer-treated WT mice was significantly suppressed compared with those from non-treated WT mice and GalCer-treated iNOS mice (Figure 6B).
A
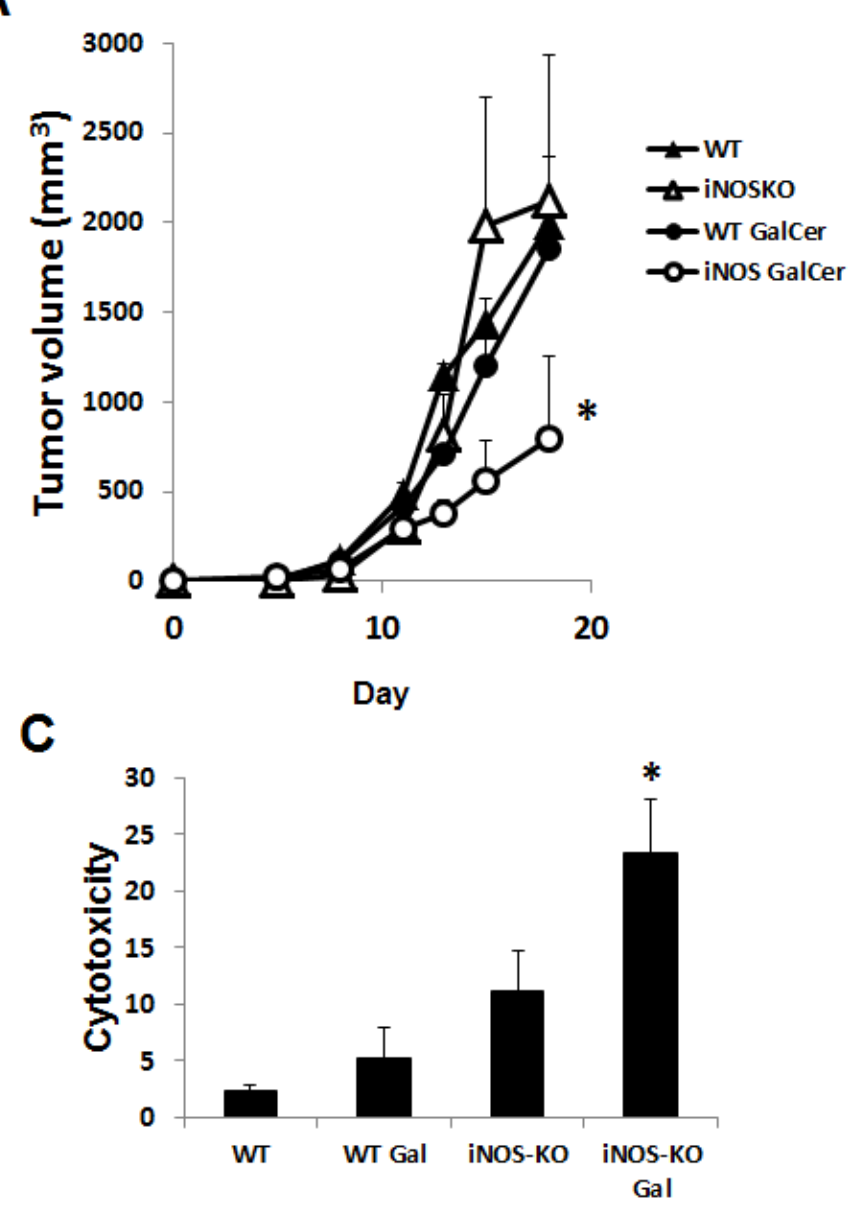

B
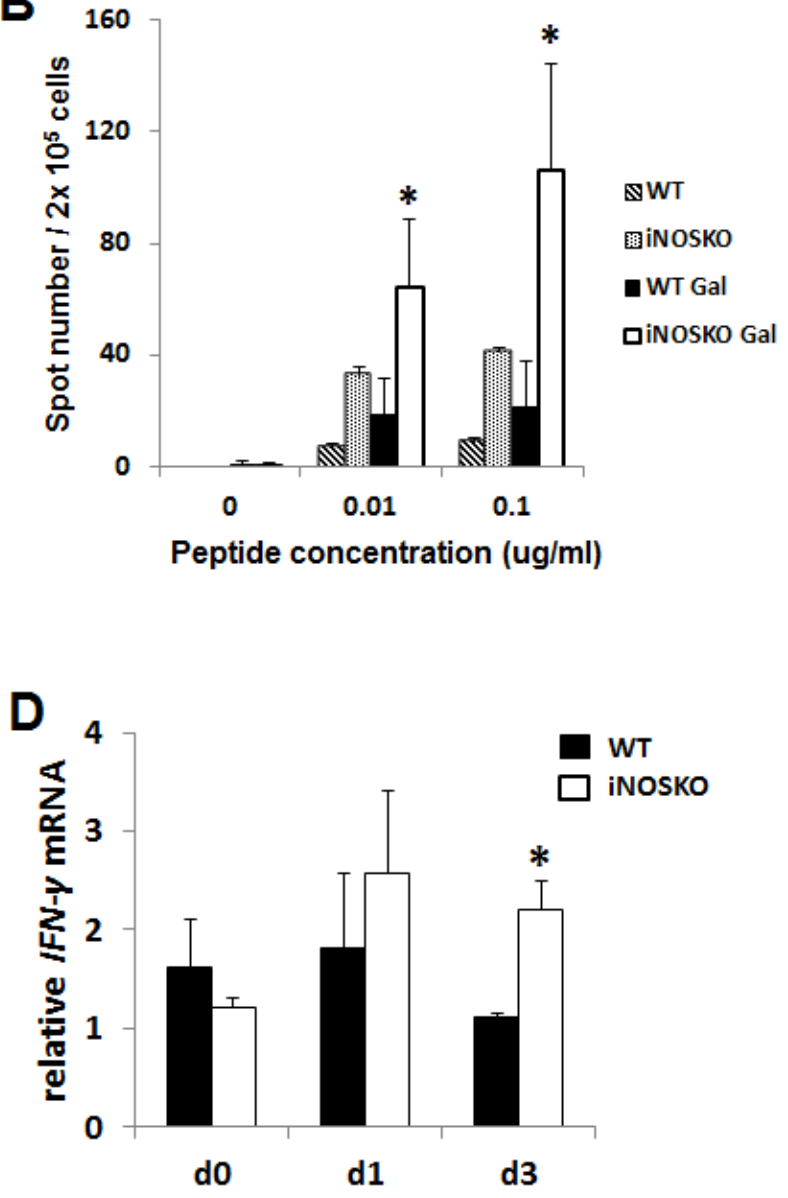

Figure 5: Inhibition of iNOS expression enhanced the anti-tumor effect of GalCer in a subcutaneous tumor model. EG7 cells $\left(1 \times 10^{6} /\right.$ mouse) were subcutaneously inoculated into the flank of WT and iNOS-KO mice on day 0 . After the tumors became palpable, the mice were intraperitoneally injected with GalCer $(2 \mu \mathrm{g} /$ mouse $)$. A. Data show the mean $\pm \mathrm{SEM}$ increase in tumor size 6-8 mice per group, from two experiments. B. DLN in tumor-bearing WT and iNOS-KO mice were isolated 7 days after GalCer intraperitoneal injection. These DLN cells were stimulated with OVA peptide in vitro and monitored for IFN- $\gamma$ production using ELISPOT assay. Results represent the mean \pm SEM of 4 mice per group. The number of spot was significantly increased in DLN cells from iNOS-KO mice treated with GalCer $(P<0.05)$. C. Isolated effector cells $(\mathrm{CD} 8+\mathrm{T}$ cells $)$ from DLN of WT and iNOS-KO mice treated with GalCer were incubated for 6 $\mathrm{h}$ with CFSE-labeled EG7 at an effector to target cell ratio of 20 to 1. Each data point and error bar represent the mean and SE, respectively, of results for triplicate samples. D. The relative expression of IFN- $\gamma$ mRNA in DLN of WT and iNOS-KO mice treated with GalCer was measured using real-time RT-PCR. The results were normalized to the expression of $18 \mathrm{~S}$ rRNA. The expression of IFN- $\gamma$ mRNA was significantly increased in iNOS-KO mice treated with GalCer $(P<0.05)$. 
A

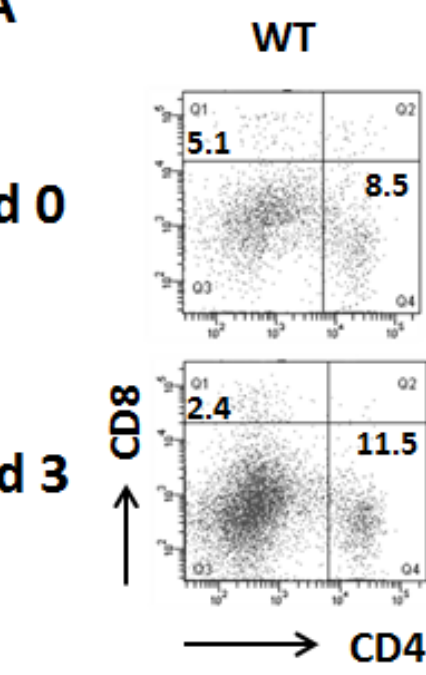

iNOS-KO

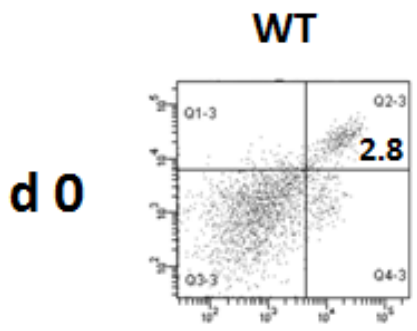

iNOS-KO
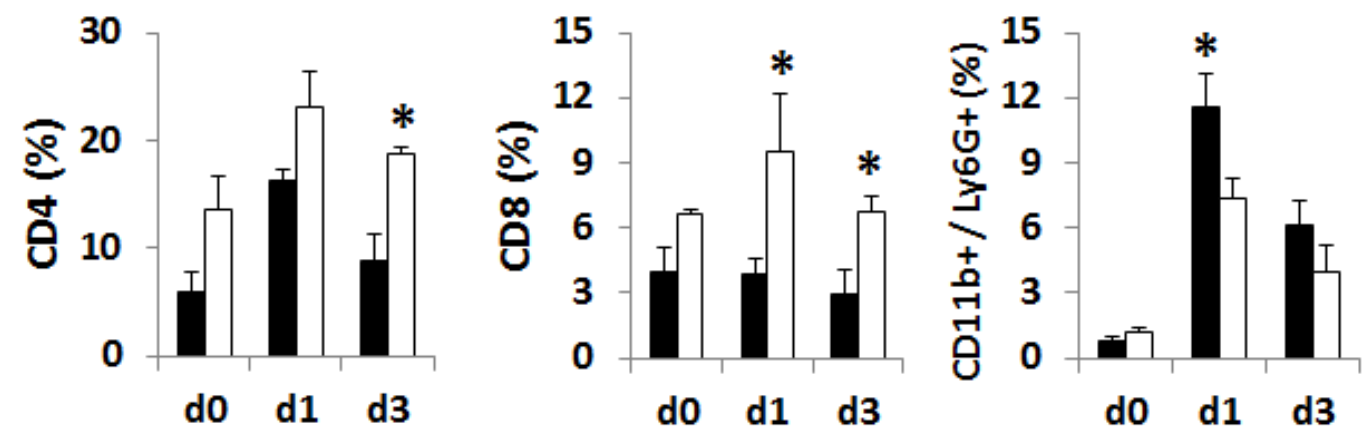

B

Non-treated

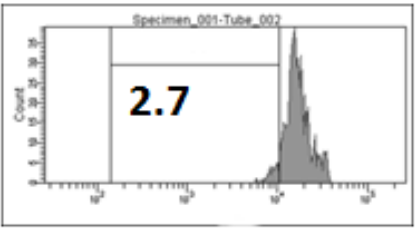

PMA

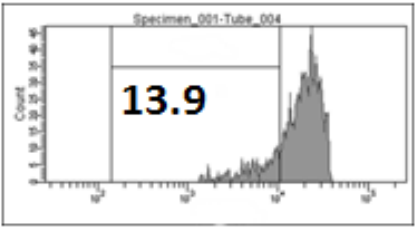

PMA + WT

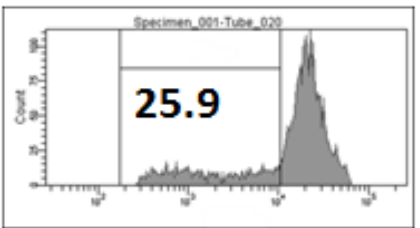

PMA + GalCer-treated WT

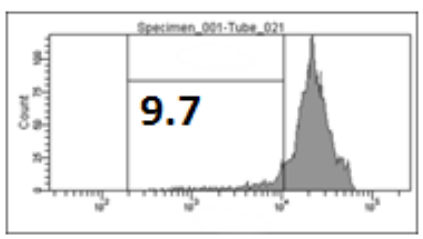

PMA + iNOSKO

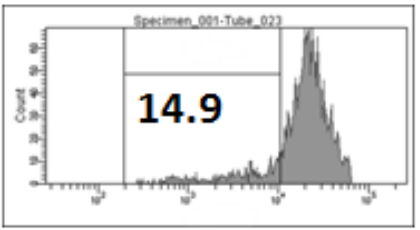

PMA + GalCer-treated iNOSKO

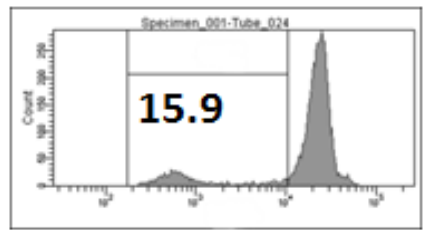

Figure 6: Changes of the proportion of CD8+ and CD11b+/Ly6G+ cells in the tumor infiltrating lymphocytes from tumor-bearing WT and iNOS-KO mice treated with GalCer. The tumor infiltrating lymphocytes from tumor-bearing WT and iNOS-KO mice were isolated 1 and 3 days after GalCer administration. A. Data show the percentage of CD4+, CD8+, and CD11b+/Ly6G+ cells. Each data point and error bar represents the mean and SEM, respectively, of data from triplicate samples. * indicates statistically significant differences. Closed bar; WT mice, open bar; iNOS-KO mice. B. Splenic CD11b+ cells were isolated from WT and iNOS-KO mice administered with GalCer. Naïve splenocytes labeled with CFSE were co-cultured with CD11b+ cells in 96-well plates at a cell-count ration of $1 / 1$ for 3 days in the presence of PMA. Data were representative of at least three independent experiments with similar result. 


\section{DISCUSSION}

In the present study, we have shown the inhibition of iNOS activity significantly enhanced the anti-tumor effect of GalCer in lung metastatic tumor model and primary subcutaneous tumor model. The administration with GalCer enhanced the tumor antigen-specific response and cytotoxic activity in the absence of iNOS expression. Moreover, the increase of CD8+ cells and the decrease of $\mathrm{CD} 11 \mathrm{~b}+/ \mathrm{Ly} 6 \mathrm{G}+$ cells within tumor might be involved in the impairment of tumor growth.

GalCer enhances host immune system via NKT cell activation. The activation of NKT cells induces the amount of Th1 cytokines, including IFN- $\gamma$ and Th2 cytokines. Many reports indicated that the increase of Th1 cytokines production lead to the induction of antitumor immunity [18]. Therefore, the anti-tumor effect of GalCer was recently evaluated in many animal and clinical studies [19-21]. It is well-known that IFN- $\gamma$ is a strong inducer of iNOS. A recent report demonstrated that the administration with GalCer also induces the expression of iNOS and enhanced the NO production [22]. Indeed, the injection of GalCer in tumor-bearing mice up-regulated the mRNA expression of iNOS in the lung (Figure 1A). In particular, iNOS expression in $\mathrm{CD} 11 \mathrm{~b}+$ cells was markedly increased after the administration with GalCer. Monoctye/macrophage expresses CD11b molecules on surface and induces iNOS by the stimulation of IFN- $\gamma$ [23]. In the present study, the iNOS expression in CD11b+ cells of BALF might be enhanced by IFN- $\gamma$ from activated NKT cells.

As shown in Figure 2, the inhibition of iNOS activity by genetically modification or the administration with L-NAME significantly enhanced the anti-tumor effect of GalCer in lung metastatic tumor model. These results indicated that the enhancement of anti-tumor response by GalCer was not dependent on the type of cancer cell line or mice. In subcutaneous tumor model, the antitumor effect of GalCer similarly increased in iNOS$\mathrm{KO}$ mice (Figure 5A). The frequency of $\mathrm{CD} 8+$ cells in BALF of iNOS-KO mice was significantly increased by the administration with GalCer compared to that of WT mice (Figure 3A). In subcutaneous tumor model, CD8+ cells within tumor also increased in iNOS-KO mice after GalCer injection (Figure 6A). It is well-known that CD8+ cells have cytotoxic activity against tumor cells and are critical in cancer immunotherapy [24, 25]. The increase of $\mathrm{CD} 8+$ cells within tumor might lead to the enhancement of anti-tumor effect of GalCer in iNOS-KO mice. In general, $\mathrm{CD} 8+\mathrm{T}$ cells are involved in anti-tumor immunity via the production of IFN- $\gamma$, FasL, and perforin [26, 27]. In the present study, the treatment of GalCer in iNOS-KO mice increased the expression of IFN- $\gamma$ and FasL in the lung (Figure4). These data supported that CD8+ T cells within tumor of tumor-bearing iNOS-KO mice increased after GalCer administration. The expression of CXCL9
mRNA was also enhanced in iNOS-KO mice treated with GalCer (Figure 4). CXCL9 is known as the T-cell chemoattractant, which is induced by IFN- $\gamma$. The upregulation of CXCL9 in the lung might be involved in the increase of CD8+ cells in BALF of tumor-bearing iNOS-mice treated with GalCer. Moreover, we found that the frequency of CD11b+/Ly6G+ cells in iNOS-KO mice treated with GalCer was lower than that in WT mice treated with GalCer (Figures 3A and 6A). MDSCs are broadly defined as $\mathrm{CD} 11 \mathrm{~b}+/ \mathrm{Ly} 6 \mathrm{G}+$ cells. Several previous reports demonstrated that MDSCs were involved in the impairment of host immune systems at tumor-bearing animals [28, 29]. Recent studies demonstrated that iNOS enhanced the recruitment and induction of functional MDSCs [30, 31]. The present data also indicated that the frequency of MDSCs in the tumor was significantly decreased in iNOS-KO mice compared to that in WT mice. Moreover, CFSE-based proliferation assay revealed that BALF cells and CD11b+ cells from GalCer-treated WT mice had an ability to suppress the proliferation of $\mathrm{T}$ cells (Figures 3B and 6B). These suppressions were reduced in BALF cells and CD11b+ cells from GalCertreated iNOS-KO mice. The absent of iNOS activity failed the induction of functional MDSCs after the treatment of GalCer. The decrease of functional MDSCs might be involved in the enhancement of anti-tumor effect in iNOSKO mice treated with GalCer.

The inhibition of iNOS activity also enhanced the anti-tumor effect in subcutaneous tumor model (Figure 5A). ELISPOT assay revealed that tumor antigen-specific immune response was significantly increased in iNOS-KO mice treated with GalCer (Figure 5B). Moreover, CD8+ cells in DLN of GalCer-treated iNOS-KO mice extremely had the tumor specific cytotoxicity. The expression of IFN- $\gamma$ in DLN also increased in iNOS-KO mice treated with GalCer (Figure 5C). These results indicated that the inhibition of iNOS expression enhanced the tumor antigen-specific immune response after the administration of GalCer. Recent study demonstrated that the inhibition of iNOS activity enhanced the anti-tumor effect of TLR7 agonist in subcutaneous tumor model [32]. Similarly, the anti-tumor effect of GalCer was enhanced by the inhibition of iNOS activity in subcutaneous tumor model. The enormous basic studies and clinical trials evaluated the anti-tumor effect in cancer immunotherapy. However, few anti-cancer therapies can completely eliminate the tumor in subcutaneous tumor model. Many studies used the agents which excessively stimulate host immune response like GalCer or TLR agonists. These agents can induce pro-inflammatory cytokines and IFNs in cancer immunotherapy. Previous reports demonstrated that these factors could induce IL-10, iNOS, and indoleamine 2,3 deoxgenase that have the immune suppressive effect [33, 34]. The induction of excessive host immune response promotes the immune suppressive factors in host. Therefore, the anti-tumor efficacy of monotherapy using 
immune stimulate agent might be reduced by the several suppressive factors induced by these agents.

In conclusion, the present study showed that the inhibition of iNOS significantly enhanced the anti-tumor effect of GalCer in lung tumor model and subcutaneous primary tumor model. The combination therapy of GalCer and iNOS inhibitor might be a new strategy for cancer immune therapy.

\section{MATERIALS AND METHODS}

\section{Mice}

Male C57BL/6J (H-2d) wild-type (WT) mice and $\mathrm{BALB} / \mathrm{c}$ mice (age, 8-10 weeks; weight; 25-30 g) were obtained from Japan SLC Inc. (Shizuoka, Japan). iNOS knockout $(\mathrm{KO})$ mice with a $\mathrm{C} 57 \mathrm{BL} / 6 \mathrm{~J}$ background were obtained from Jackson Laboratory (Bar Harbor, ME). All procedures were conducted in accordance with the National Institutes of Health Guide for the Care and Use of Laboratory Animals, and with the guidelines for the care and use of animals established by the Animal Care and Use Committee of Gifu University.

\section{Cell lines and reagents}

The B16-F10 melanoma cell line, colon carcinoma CT26 cells, EG7 cells used in this study were generously provided by Hidekazu Shirota (Laboratory of Experimental Immunology, Cancer and Inflammation Program, National Cancer Institute, Frederick, MD). These cells were cultured in suspension in RPMI 1640 (Invitrogen Ltd., Paisley, United Kingdom) containing $10 \%$ heat inactivated FCS (PAA Laboratories, GmbH, Linz, Austria), 2 mM L-glutamine, penicillin (100 units/ $\mathrm{ml})$, and streptomycin $(100 \mathrm{~g} / \mathrm{ml}) . \mathrm{N}^{\mathrm{G}}$-nitro-l-arginine methyl ester (L-NAME) was purchased from SIGMA (St. Louis, MO). WT mice were administered with L-NAME orally at $2 \mathrm{mg} / \mathrm{ml}$ in drinking water for for 1 days before and 7 days after GalCer injection.

\section{In vivo tumor studies}

Mice were intravenously injected with B16 F10 cells $\left(3 \times 10^{5}\right.$ in C57BL/6 mice), and CT26 carcinoma cells $(2 \times$ $10^{5}$ CT26 carcinoma cells in BALB/c mice), and GalCer ( $2 \mu \mathrm{g} /$ mouse) was administered into tumor-bearing mice 7 days after the inoculation of tumor cells. Seven days after GalCer injection, mice were killed, and their lungs were removed to count superficial metastatic nodules.

To establish the subcutaneous tumor model, mice were challenged with EG7-OVA cells $\left(1 \times 10^{6}\right.$ cells in C57BL/6 mice). This tumor cell lines formed solid tumors when the tumor cells were subcutaneously inoculated into the flank. Tumor cells were implanted into the flank of WT or iNOS-KO mice on day 0. After the tumors became palpable ( $>5 \mathrm{~mm}$ in diameter), the mice were intraperitoneally administered with GalCer ( $2 \mu \mathrm{g} / \mathrm{mouse})$. Tumor growth curves were generated using 6-9 mice per group, and all results were derived by combining data from 2 to 3 independent experiments. As previous study, tumor size was calculated using the following formula: (length $\times$ width $\times$ height) $/ 2$.

\section{ELISPOT assay}

The ELISPOT assay was performed as described previously [35]. Single-cell suspensions were prepared from the draining lymph node (DLN) of subcutaneous tumor-bearing mice treated with GalCer. A total of $2.0 \times$ $10^{5}$ cells per well were stimulated for $14-16 \mathrm{~h}$ with $0,0.1$, or $1 \mu \mathrm{g} / \mathrm{ml}$ of ova SIINFEKL peptide in 96 well MultiScreen filter plates (Millipore, Billerica, MA) previously coated with monoclonal rat anti-IFN- $\gamma$ antibody (Ab) (clone; R46A2) (BD Biosciences, San Jose, CA). The plates were washed and treated with biotinylated polyclonal goat antiIFN- $\gamma$ Ab (R\&D Systems, Minneapolis, MN) followed by streptavidin alkaline phosphatase. Staining was visualized thorough the addition of a 5-bromo-4-chloro-3-indolyl phosphatase solution (Sigma-Aldrich, St. Louis, MO) and counted manually under $40 \times$ magnification. A single-blind reviewer counted the number of cytokine-secreting cells, and all data were generated by analyzing three separate wells per sample.

\section{Real-time reverse transcription-PCR}

Total RNA was isolated from lung tissue, BALF cells and tumor tissue, and transcribed into complementary DNA (cDNA) using an RNeasy Mini Kit (QIAGEN, Hilden, Germany) and a high capacity cDNA transcription kit (Applied Biosystems, Foster City, CA). The resulting cDNA was used as a template for real-time RT-PCR along with primer-probe sets for iNOS, CCL2, CXCL9, FasL, IFN- $\gamma$, and 18S (TaqMan Gene Expression Assays; Applied Biosystems) and TaqMan universal PCR master mix (Applied Biosystems) according to the manufacturer's recommendations. 18S rRNA was used. Real-time RTPCR was carried out using a Light-Cycler 480 system (Roche Diagnostic Systems, Basel, Switzerland). The calculation of relative gene expression differences was done by the comparative $2-\Delta \Delta \mathrm{CT}$ method.

\section{Flow cytometric analysis}

BALF cells and intra-tumor lymphocytes were isolated from tumor-bearing mice treated with GalCer. 
Cell viability and number were assessed by trypan blue exclusion. For flow cytometry, $2 \times 10^{5}$ cells were stained using a standard protocol. The following antibodies (Abs) were used: APC-labeled anti-mouse CD4 mAb (clone GK1.5; eBiosciences, San Diego, CA), PE-Cy7-labeled anti-mouse CD8 mAb (clone 53-6.7; eBiosciences, San Diego, CA), PE-Cy7-labeled anti-mouse CD11b mAb (clone M1/70; eBiosciences), and FITC-labeled antimouse Ly-6G mAb (clone RB6-8C5; BD Biosciences). Samples were acquired using a FACSCanto 11 flow cytometer and data analysis was performed using FACSDiva software (BD Biosciences).

\section{Isolation of CD8+ and CD11b+ cells}

CD8+ cells in DLN were isolated by magnetic beads conjugated with an anti-CD8 antibody (Miltenyi Biotec) as described in our previous report [36]. BALF cells were sorted by $\mathrm{CD} 11 \mathrm{~b}+$ status using magnetic beads conjugated with an anti-CD11b antibody (Miltenyi Biotec). The magnetically labeled cells were purified using quadroMACS system (Miltenyi Biotec).

\section{Cytotoxicity assay}

The cytolytic activity of EG7-specific lymphocytes was assessed using a fluorescent-based dye, 5- (and 6-) carboxyfluorescein diacetate succinimydyl ester (CFSE) as described previously [25] . Target EG7 cells were labeled with CFSE as follows: cells were suspended in PBS and concentrated to $1 \times 106 / \mathrm{ml}$. For targets cells, $0.5 \mu \mathrm{l}$ of CFSE stock solution $(5 \mathrm{mM})$ was added to $1 \mathrm{ml}$ of cell suspension and incubated for $4 \mathrm{~min}$ at room temperature. For control targets cells, $0.5 \mu \mathrm{l}$ of diluted CFSE solution $(100 \mu \mathrm{M})$ was used for labeling. Labeled targets and various numbers of effector cells were added to a final volume of $200 \mu \mathrm{l}$ in each well of the 96 -well round-bottom plates and incubated for $6 \mathrm{~h}$ at $37^{\circ} \mathrm{C}$. After incubation, sensitive target cells were mixed with control cells in a tube with PBS containing $1 \%$ FCS and $0.1 \%$ sodium azide. The mixed cells were washed once and suspended in $4 \%$ paraformaldehyde-containing PBS and stored at $4^{\circ} \mathrm{C}$ in the dark until cytometer acquisition. Acquisition was performed using a FACSCantoII (BD Immunocytometry Systems). All samples were assayed in duplicate and the mean percentage of specific lysis was calculated as follows: \% specific lysis $=[$ (number of sensitive target cells in control sample - number of sensitive target cells in test sample) / number of sensitive target cells in control sample] $\times 100$. The terms control sample and test sample indicate target cells incubated without added effector cells and target cells incubated with added effector cells, respectively.

\section{In vitro CFSE proliferation assay}

BALF cells and isolated CD11b + splenocytes were co-cultured with PMA-stimulated CD3+ cells in CFSEbased proliferation assay. Splenoctyes were labeled with CFSE as previously described. CFSE-labeled cells were co-cultured with BALF cells or isolated CD11b + cells from GalCer-treated mice in 96-well plates for 3 days at $37^{\circ} \mathrm{C}$ in a $5 \% \mathrm{CO} 2$ atmosphere. To induce proliferation, CFSE-labeled cells were stimulated with PMA. CFSElabeled splenocytes were analyzed by flow cytometer.

\section{Statistics}

Values are expressed as means \pm standard errors of the mean (SEMs). Differences between experimental and control groups were analyzed by the Kruskal-Wallis test followed by Scheffe's F-test. The Wilcoxon's test of Kaplan-Meier plots was used to analyze differences in animal survival. Significance was established at $P<0.05$.

\section{ACKNOWLEDGMENTS}

The authors thank Dr. H. Shirota for kindly providing several of the tumor cell lines in this study. The authors would like to thank Editage (http://www.editage. jp) for the English language review.

\section{GRANT SUPPORT}

This work was supported by Grant-in-Aid for Scientific Research (C) (25460679) from the Ministry for Education, Culture, Sports, Science and Technology of Japan.

\section{CONFLICTS OF INTEREST}

The authors declare no competing financial interests.

\section{REFERENCES}

1. Terabe M and Berzofsky JA. The immunoregulatory role of type I and type II NKT cells in cancer and other diseases. Cancer immunology, immunotherapy : CII. 2014; 63:199213.

2. Ito H, Ando K, Nakayama T, Taniguchi M, Ezaki T, Saito K, Takemura M, Sekikawa K, Imawari M, Seishima $\mathrm{M}$ and Moriwaki H. Role of Valpha 14 NKT cells in the development of impaired liver regeneration in vivo. Hepatology. 2003; 38:1116-1124.

3. Ito H, Koide N, Hassan F, Islam S, Tumurkhuu G, Mori I, Yoshida T, Kakumu S, Moriwaki H and Yokochi T. Lethal endotoxic shock using alpha-galactosylceramide sensitization as a new experimental model of septic shock. 
Lab Invest. 2006; 86:254-261.

4. Ito $\mathrm{H}$, Ando $\mathrm{K}$, Ishikawa $\mathrm{T}$, Nakayama $\mathrm{T}$, Taniguchi $\mathrm{M}$, Saito K, Imawari M, Moriwaki H, Yokochi T, Kakumu $\mathrm{S}$ and Seishima M. Role of Valpha14+ NKT cells in the development of Hepatitis B virus-specific CTL: activation of Valpha14+ NKT cells promotes the breakage of CTL tolerance. International immunology. 2008; 20:869-879.

5. Fuji N, Ueda Y, Fujiwara H, Toh T, Yoshimura $\mathrm{T}$ and Yamagishi H. Antitumor effect of alpha-galactosylceramide (KRN7000) on spontaneous hepatic metastases requires endogenous interleukin 12 in the liver. Clinical cancer research : an official journal of the American Association for Cancer Research. 2000; 6:3380-3387.

6. Smyth MJ, Crowe NY, Pellicci DG, Kyparissoudis K, Kelly JM, Takeda K, Yagita H and Godfrey DI. Sequential production of interferon-gamma by NK1.1(+) T cells and natural killer cells is essential for the antimetastatic effect of alpha-galactosylceramide. Blood. 2002; 99:1259-1266.

7. Nakui M, Ohta A, Sekimoto M, Sato M, Iwakabe K, Yahata T, Kitamura H, Koda T, Kawano T, Makuuchi H, Taniguchi $\mathrm{M}$ and Nishimura T. Potentiation of antitumor effect of NKT cell ligand, alpha-galactosylceramide by combination with IL-12 on lung metastasis of malignant melanoma cells. Clinical \& experimental metastasis. 2000; 18:147-153.

8. Nishio S, Yamada N, Ohyama H, Yamanegi K, Nakasho K, Hata M, Nakamura Y, Fukunaga S, Futani H, Yoshiya S, Ueda H, Taniguchi M, Okamura H and Terada N. Enhanced suppression of pulmonary metastasis of malignant melanoma cells by combined administration of alphagalactosylceramide and interleukin-18. Cancer science. 2008; 99:113-120.

9. Xu W, Liu LZ, Loizidou M, Ahmed M and Charles IG. The role of nitric oxide in cancer. Cell Res. 2002; 12:311-320.

10. Lagares-Garcia JA, Moore RA, Collier B, Heggere M, Diaz F and Qian F. Nitric oxide synthase as a marker in colorectal carcinoma. Am Surg. 2001; 67:709-713.

11. Thomsen LL, Miles DW, Happerfield L, Bobrow LG, Knowles RG and Moncada S. Nitric oxide synthase activity in human breast cancer. Br J Cancer. 1995; 72:41-44.

12. Ochoa AC, Zea AH, Hernandez $\mathrm{C}$ and Rodriguez PC. Arginase, prostaglandins, and myeloid-derived suppressor cells in renal cell carcinoma. Clin Cancer Res. 2007; 13:721s-726s.

13. Parekh VV, Wu L, Olivares-Villagomez D, Wilson KT and Van Kaer L. Activated invariant NKT cells control central nervous system autoimmunity in a mechanism that involves myeloid-derived suppressor cells. J Immunol. 2013; 190:1948-1960.

14. Ito H, Koide N, Morikawa A, Hassan F, Islam S, Tumurkhuu G, Mori I, Yoshida T, Kakumu S, Moriwaki H and Yokochi T. Augmentation of lipopolysaccharide-induced nitric oxide production by alpha-galactosylceramide in mouse peritoneal cells. J Endotoxin Res. 2005; 11:213-219.

15. Ohtaki H, Ito H, Ando K, Ishikawa T, Saito K, Imawari
M, Yokochi T, Moriwaki H and Seishima M. Valpha14 NKT cells activated by alpha-galactosylceramide augment lipopolysaccharide-induced nitric oxide production in mouse intra-hepatic lymphocytes. Biochemical and biophysical research communications. 2009; 378:579-583.

16. Ji Z, Fan Z, Zhang Y, Yu R, Yang H, Zhou C, Luo J and Ke ZJ. Thiamine deficiency promotes $\mathrm{T}$ cell infiltration in experimental autoimmune encephalomyelitis: the involvement of CCL2. J Immunol. 2014; 193:2157-2167.

17. Ochiai E, Sa Q, Brogli M, Kudo T, Wang X, Dubey JP and Suzuki Y. CXCL9 is important for recruiting immune $\mathrm{T}$ cells into the brain and inducing an accumulation of the $\mathrm{T}$ cells to the areas of tachyzoite proliferation to prevent reactivation of chronic cerebral infection with Toxoplasma gondii. Am J Pathol. 2015; 185:314-324.

18. Colombo MP and Trinchieri G. Interleukin-12 in anti-tumor immunity and immunotherapy. Cytokine Growth Factor Rev. 2002; 13:155-168.

19. Ando $\mathrm{T}$, Ito $\mathrm{H}$, Arioka $\mathrm{Y}$, Ogiso $\mathrm{H}$ and Seishima M. Combination therapy with alpha-galactosylceramide and a Toll-like receptor agonist exerts an augmented suppressive effect on lung tumor metastasis in a mouse model. Oncology reports. 2015; 33:826-832.

20. Macho-Fernandez E, Cruz LJ, Ghinnagow R, Fontaine J, Bialecki E, Frisch B, Trottein F and Faveeuw C. Targeted delivery of alpha-galactosylceramide to CD8alpha+ dendritic cells optimizes type I NKT cell-based antitumor responses. J Immunol. 2014; 193:961-969.

21. Nicol AJ, Tazbirkova A and Nieda M. Comparison of clinical and immunological effects of intravenous and intradermal administration of alpha-galactosylceramide (KRN7000)-pulsed dendritic cells. Clin Cancer Res. 2011; 17:5140-5151.

22. Jeong YH, Kim Y, Song H, Chung YS, Park SB and Kim HS. Anti-inflammatory effects of alpha-galactosylceramide analogs in activated microglia: involvement of the p38 MAPK signaling pathway. PloS one. 2014; 9:e87030.

23. Riquelme P, Tomiuk S, Kammler A, Fandrich F, Schlitt HJ, Geissler EK and Hutchinson JA. IFN-gamma-induced iNOS expression in mouse regulatory macrophages prolongs allograft survival in fully immunocompetent recipients. Mol Ther. 2013; 21:409-422.

24. McNamara MJ, Kasiewicz MJ, Linch SN, Dubay C and Redmond WL. Common gamma chain (gammac) cytokines differentially potentiate TNFR family signaling in antigenactivated CD8(+) T cells. J Immunother Cancer. 2014; 2:28.

25. Ito $\mathrm{H}$, Ando $\mathrm{T}$, Arioka $\mathrm{Y}$, Saito $\mathrm{K}$ and Seishima $\mathrm{M}$. Inhibition of indoleamine 2,3-dioxygenase activity enhances the anti-tumour effects of a Toll-like receptor 7 agonist in an established cancer model. Immunology. 2015; 144:621-630.

26. Ramirez-Montagut T, Chow A, Kochman AA, Smith OM, Suh D, Sindhi H, Lu S, Borsotti C, Grubin J, Patel N, Terwey TH, Kim TD, Heller G, Murphy GF, Liu 
C, Alpdogan O, et al. IFN-gamma and Fas ligand are required for graft-versus-tumor activity against renal cell carcinoma in the absence of lethal graft-versus-host disease. J Immunol. 2007; 179:1669-1680.

27. Su MW, Pyarajan S, Chang JH, Yu CL, Jin YJ, Stierhof YD, Walden P and Burakoff SJ. Fratricide of CD8+ cytotoxic $\mathrm{T}$ lymphocytes is dependent on cellular activation and perforin-mediated killing. Eur J Immunol. 2004; 34:24592470.

28. Khaled YS, Ammori BJ and Elkord E. Myeloid-derived suppressor cells in cancer: recent progress and prospects. Immunol Cell Biol. 2013; 91:493-502.

29. Ostrand-Rosenberg S and Sinha P. Myeloid-derived suppressor cells: linking inflammation and cancer. J Immunol. 2009; 182:4499-4506.

30. Zhang Y, Bi Y, Yang H, Chen X, Liu H, Lu Y, Zhang Z, Liao J, Yang S, Chu Y, Yang R and Liu G. mTOR limits the recruitment of $\mathrm{CD} 11 \mathrm{~b}+\mathrm{Gr} 1+\mathrm{Ly} 6 \mathrm{Chigh}$ myeloid-derived suppressor cells in protecting against murine immunological hepatic injury. J Leukoc Biol. 2014; 95:961-970.

31. Liu G, Bi Y, Wang R, Yang H, Zhang Y, Wang X, Liu H, Lu Y, Zhang Z, Chen W, Chu Y and Yang R. Targeting S1P1 receptor protects against murine immunological hepatic injury through myeloid-derived suppressor cells. J Immunol. 2014; 192:3068-3079.

32. Ito $\mathrm{H}$, Ando $\mathrm{T}$, Ogiso $\mathrm{H}$, Arioka $\mathrm{Y}$ and Seishima $\mathrm{M}$. Inhibition of induced nitric oxide synthase enhances the anti-tumor effects on cancer immunotherapy using TLR7 agonist in mice. Cancer immunology, immunotherapy : CII. 2015; 64:429-436.

33. Iwamoto N, Ito H, Ando K, Ishikawa T, Hara A, Taguchi A, Saito K, Takemura M, Imawari M, Moriwaki H and Seishima M. Upregulation of indoleamine 2,3-dioxygenase in hepatocyte during acute hepatitis caused by hepatitis $\mathrm{B}$ virus-specific cytotoxic $\mathrm{T}$ lymphocytes in vivo. Liver international : official journal of the International Association for the Study of the Liver. 2009; 29:277-283.

34. Elcombe SE, Naqvi S, Van Den Bosch MW, MacKenzie KF, Cianfanelli F, Brown GD and Arthur JS. Dectin-1 regulates IL-10 production via a MSK1/2 and CREB dependent pathway and promotes the induction of regulatory macrophage markers. PloS one. 2013; 8:e60086.

35. Ito H, Ando T, Ando K, Ishikawa T, Saito K, Moriwaki $\mathrm{H}$ and Seishima M. Induction of HBsAg-specific cytotoxic $\mathrm{T}$ lymphocytes can be up-regulated by the inhibition of indoleamine 2, 3-dioxygenase activity. Immunology. 2014; 142:614-23.

36. Ito $\mathrm{H}$, Ando $\mathrm{K}$, Ishikawa $\mathrm{T}$, Saito $\mathrm{K}$, Takemura $\mathrm{M}$, Imawari M, Moriwaki $\mathrm{H}$ and Seishima M. Role of TNFalpha produced by nonantigen-specific cells in a fulminant hepatitis mouse model. J Immunol. 2009; 182:391-397. 